\title{
La difficile mise en place des districts sanitaires urbains
}

Eric Gauvrit et Raphaël Okalla

\section{(2) OpenEdition \\ Journals}

Édition électronique

URL : http://journals.openedition.org/apad/186

DOI : $10.4000 /$ apad. 186

ISSN : 1950-6929

Éditeur

LIT Verlag

Édition imprimée

Date de publication : 1 juin 2001

Référence électronique

Eric Gauvrit et Raphaël Okalla, "La diffıcile mise en place des districts sanitaires urbains », Bulletin de I'APAD [En ligne], 21 | 2001, mis en ligne le 06 mars 2006, consulté le 07 septembre 2020. URL : http:// journals.openedition.org/apad/186 ; DOI : https://doi.org/10.4000/apad.186

Ce document a été généré automatiquement le 7 septembre 2020

Bulletin de l'APAD 


\title{
La difficile mise en place des districts sanitaires urbains
}

\author{
Eric Gauvrit et Raphaël Okalla
}

1 Figures emblématiques des politiques définies par l'Organisation Mondiale de la Santé (OMS) depuis la conférence d'Harare en 1987, les districts de santé se sont mis en place au Cameroun dans le cadre global de la politique dite de "Réorganisation des Soins de Santé Primaires" (REO des SSP). Ils ont conduit à la confection d'une nouvelle carte sanitaire dans ce pays tout au long des années 1990. Cette carte va se trouver écartelée entre de multiples contraintes politiques, administratives et sanitaires, contraintes exacerbées par la mise en place concomitante d'une ouverture politique et de la décentralisation.

2 Une illustration de tous ces enjeux peut être fourni par l'implantation de districts de santé dans un cadre urbain avec l'exemple particulier de celui de Biyem Assi, district-pilote de la capitale camerounaise, Yaoundé.

La délimitation des districts de santé au Cameroun

3 Dans le cadre de la mise en place des districts de santé, plusieurs critères ont été élaborés. Il s'agit des critères de la démographie, de l'accessibilité géographique, de l'affinité socio-culturelle des populations, de la disponibilité des structures sanitaires existantes, de la potentialité économique de la zone donnée et de la conformité avec le découpage administratif. Dans l'application de ces critères, des distorsions vont apparaître. Elles résulteront, pour partie, de la pluralité des acteurs qui ont été amenés à intervenir pour élaborer et exécuter la carte sanitaire camerounaise, chaque acteur venant avec sa philosophie en matière de santé, ses méthodes, ses approches, ses attentes. Ce manque de coordination proviendra également de l'absence d'un canevas précis de politique sanitaire nationale. Ce sera tout l'enjeu de la Réorganisation des Soins de Santé Primaires (REO des SSP) que de coordonner toutes ces actions autour d'un axe fédérateur, reconnu et partagé par tous : le Plan de Développement Sanitaire.

4 Le moyen d'y aboutir sera fourni par une série de séminaires et d'ateliers au début des années 1990 dans le cadre d'une vaste concertation de tous les intervenants dans le domaine de la santé. Plusieurs ateliers ont été organisés à Bertoua, Bafoussam, Kribi, et 
ont conduit à un éclairage parcellisé de la réforme; les différents séminaires abordaient des questions distinctes: à Bertoua, la carte sanitaire; à Bafoussam, les normes à établir pour les hôpitaux de district; d'autres séminaires envisageaient la question des normes en équipements et en personnels.

5 Au cours de cette concertation, des logiques opposées vont se dessiner. Le Ministère de la santé (les Délégués provinciaux au niveau local, la Direction de la Médecine Préventive au niveau central) entendait privilégier une flexibilité des critères pour une meilleure logique d'opérationnalité des districts de santé. La délimitation de ces derniers ne devait pas forcément suivre les critères démographiques et de conformité avec le découpage administratif. Or, les autres acteurs de ce découpage entendaient, eux, axer leurs actions sur deux logiques d'intervention différentes :

- accorder la primauté à la conformité au découpage administratif existant, pour le personnel politique local

- accorder la primauté au critère démographique, pour les bailleurs de fonds œuvrant dans le domaine de la santé

- une source de conflit dans le découpage de la carte sanitaire est donc apparu à la suite de la forte attente des acteurs politiques locaux à l'égard de cette délimitation territoriale.

La solution prônée par les représentants politiques lors de la concertation nationale à Bertoua en 1992 était de faire coïncider le district de santé avec l'arrondissement administratif ${ }^{1}$, chaque leader politique local souhaitant peu ou prou "bénéficier" de "son" district de santé. Cette volonté était d'autant plus forte que l'Administration venait de créer de nouveaux arrondissements dans le pays. Ce processus de scissiparité administratif avait conduit à un besoin d'affirmation identitaire pour les nouveaux venus. Une des modalités de cette affirmation pouvait passer par la création d'un district de santé, et des structures afférentes (hôpital, centres de santé). Car un hôpital de district, par exemple, "donnait à voir" le nouvel arrondissement, l'établissait dans le paysage administratif camerounais. Sa présence, son équipement, voire son sur-équipement était également un révélateur de la puissance de l'élite politique ou administrative locale.

7 D'autre part, pour le personnel politique, le besoin de posséder "son" hôpital de district était renforcé par la confusion qui demeurait entre ces nouveaux hôpitaux et les hôpitaux départementaux qui structuraient l'ancien paysage sanitaire du pays. Les hommes politiques locaux se demandaient parfois "si l'arrondissement dans lequel on était en train d'implanter le nouvel hôpital de district n'allait pas devenir à terme le chef-lieu d'un prochain département", avec toute l'élévation politique subséquente pour l'élite locale concernée.

Une confusion existait également pour le terme même de district. Au Cameroun, le district correspond initialement à une unité administrative plus petite que l'arrondissement. Il n'est présent que dans certaines parties du territoire camerounais, où l'enclavement nécessite une subdivision supplémentaire pour les actes administratifs les plus courants. C'est donc cette structure administrative marginale, placée sous l'autorité de la sous-préfecture, qui fait sens lorsque est évoqué le mot district. La confusion est donc possible entre le district administratif et le district sanitaire pour la majeure partie de la population, personnel politique compris. Et ce d'autant plus que la notion de district sanitaire n'est pas encore une réalité palpable pour beaucoup de personnes. Ainsi, par exemple, le district de santé de Biyem Assi à Yaoundé fera plus référence par le terme de Biyem Assi à un quartier particulier de 
Yaoundé VI qu'à un ensemble sanitaire géographiquement constitué; et un habitant des quartiers d'Etoa ou d'Efoulan (intégrés au district de Biyem Assi) ne se reconnaîtra pas spontanément comme résidant dans le district sanitaire de Biyem Assi.

Pour les hauts fonctionnaires du ministère de la Santé le découpage sanitaire passait plutôt par deux voies :

- Faire en sorte qu'un hôpital de district puisse desservir plusieurs arrondissements, si les critères retenus comme la situation démographique, sanitaire, etc., l'exigeaient. Une telle démarche induisait une abondance de précautions oratoires. Pour le Directeur de la Médecine Préventive de cette époque, il était politiquement impossible de dire, par exemple, que l'arrondissement de Mban serait rattaché à celui de Batouri, où se situerait l'hôpital de district. Politiquement, il n'était pas possible, pour les élites locales, d'accepter qu'un arrondissement soit rattaché à un autre. Il fallait spécifier que l'hôpital construit à Batouri desservirait les arrondissements untel et untel.

- Sortir de la carte administrative, le cas échéant, pour tenter de viabiliser un district de santé, au regard des bassins de fréquentation des hôpitaux déjà existants.

C'est ainsi que le constat avait été fait par le ministère que les hôpitaux départementaux qui fonctionnaient déjà, desservaient principalement la population qui était dans le chef-lieu. Ensuite son bassin de fréquentation tenait plus des contingences géographiques (distance, facilité de déplacement) ou sanitaires (qualité $\mathrm{du}$ plateau technique, tissu des formations sanitaires publiques ou privées/ confessionnelles présentes), que des délimitations administratives.

11 Cette démarche d'affranchissement de la structuration administrative était renforcée par les bailleurs de fonds qui souhaitaient conforter leur "politique du projet accompli". Ainsi, initialement présents au Cameroun pour des projets de développement sanitaire, rural, d'aménagement urbain ou routier, des agences de coopération ont souhaité adjoindre un volet santé ou préserver les acquis du volet santé initial ; les bailleurs entendaient faire pression, le cas échéant, sur le Ministère de la santé pour que la zone aidée devienne un district, que cette zone corresponde à une partie d'arrondissement, à un arrondissement entier ou bien à une province. La logique d'un découpage sanitaire selon une base territoriale pré-établie se trouvait ainsi confrontée à une logique de puissance financière déséquilibrée entre la puissance publique et les bailleurs de fonds, un bailleur pouvant amener plus d'argent pour "son" seul district d'intervention que l'Etat pour toute la province.

Par ailleurs, les bailleurs de fonds s'opposèrent aux cadres du Ministère de la santé, concepteurs du projet de découpage des districts de santé, sur la question du critère démographique. Ce critère fut particulièrement mis en avant par les bailleurs de fonds lors de l'atelier de Bertoua. En filigrane, ce critère indique qu'il faut un seuil critique de population par district pour que celui-ci soit viable. Un district doit, dans ces conditions, englober une population entre 100.000 et 200.000 habitants. Chaque district doit pouvoir être découpé en 7 à 10 aires de santé pour les districts ruraux, et 10 à 15 pour les districts urbains. Cet impératif démographique prôné par les bailleurs va se heurter à la structuration administrative et démographique du Cameroun. Car s'il est apparu rapidement peu viable d'implanter un district de santé par arrondissement, le niveau du département a semblé, quant à lui, incontournable pour les responsables nationaux et les Délégués provinciaux de la Santé. Or, il existe des départements qui ne regroupent pas 100.000 habitants, notamment dans les provinces de l'Est, de l'Adamaoua et d'une partie du Centre. Pour ces provinces, la faible densité 
démographique a conduit les Délégués à élaborer des districts de 27.000 habitants, par exemple, pour ne pas à avoir à se retrouver avec des districts d'une superficie de plusieurs dizaines de kilomètres. Ces propositions de découpage ont rencontré l'opposition de plusieurs bailleurs de fonds qui ont contesté la viabilité de tels districts. Les négociations entamées entre le niveau central du ministère et les bailleurs réticents ont permis d'abaisser le seuil démographique minimum pour un district de 100.000 à moins de 40.000 habitants, à condition de considérer ces districts comme expérimentaux et non-prioritaires pour les interventions extérieures. Étant donné la faible capacité financière de l'Etat, on peut se demander si cela n'a pas été une victoire à la Pyrrhus pour l'Administration centrale camerounaise.

13 Car ces négociations se sont déroulées dans un contexte de crises tant politique qu'économique qui ont particulièrement fragilisé l'Etat camerounais dans son rôle d'instance décisionnaire et de coordination. Sur le plan économique et financier, "le pays était à genoux financièrement lorsque la réforme a commencé. Aussi, par rapport à la capacité financière du gouvernement, les bailleurs ont pu imposer leurs points de vue", à travers ce que le responsable de la Santé communautaire considère comme des conditionnalités. Les forces centrifuges des bailleurs de fonds ont distendu la carte sanitaire à leur profit. Cette prédominance financière leur donnait en effet une considérable latitude d'action et de décision, face à un Etat à la marge de manœuvre réduite et qui ne pouvait plus jouer son rôle de régulateur qu'il détenait au départ de la réforme.

Il convient en outre de souligner qu'un angle mort de la carte sanitaire camerounaise a pu être constitué par l'insuffisance de la prise en compte des formations privées confessionnelles, tout particulièrement en milieu urbain. Or, $40 \%$ des actes de soins dispensé au Cameroun le sont par le secteur confessionnel; le taux de fréquentation des structures publiques s'élève à 0,3 contacts par an et par habitant, contre 0,9 pour le secteur confessionnel. Cette insuffisance de prise en compte a pu conduire à des situations telle que la rénovation d'un hôpital de district par une agence multilatérale ne se traduisant pas par une augmentation de ]a fréquentation, car l'hôpital réhabilité était concurrencé par une structure confessionnelle à proximité qui répondait déjà aux attentes des malades-usagers.

Les districts urbains de Yaoundé

15 Les bailleurs de fonds ont, dans un premier temps, considéré les Soins de Santé Primaires (SSP) et l'implantation des districts de santé - clés de voûte de la réforme comme les modalités organisationnelles du système de santé en milieu rural uniquement. Les zones urbaines n'étaient pas exclues stricto sensu du champ d'application des réformes sanitaires, mais étaient délaissées par les bailleurs qui préféraient se concentrer sur les zones rurales. Les villes étaient laissées à leur structuration hospitalo-centrée. La nécessité d'étendre de façon globale la nouvelle carte sanitaire a conduit le Cameroun à élaborer un projet-pilote et à faire appel en 1991 à un consultant pour l'édification des normes et des recommandations permettant l'application des SSP en zone urbaine. Sur la base de ce document, le gouvernement a demandé un financement auprès de la Banque Mondiale pour implanter les districts de santé en zone urbaine, et tout particulièrement dans les deux principales villes du pays : Yaoundé et Douala.

Le découpage a été réalisé initialement par les experts du ministère de la Santé qui ont travaillé sur la mise en place de la politique du district de santé au Cameroun. Le 
principe retenu était de se baser sur le découpage administratif pré-existant. La délimitation des districts urbains de Yaoundé et Douala date ainsi de 1991 pour les études et 1992 pour les réalisations. De l'avis des experts, la ville semble plus complexe parce qu'il n'y a pas de "délimitations naturelles", comme les cours d'eau par exemple. La délimitation géographiques des districts de santé peut devenir un enjeu de pouvoir et de compétition. Aussi, tout travail de délimitation doit se faire par la négociation pour inclure ou non tel quartier ou tel village de la communauté urbaine dans un district sanitaire particulier. Les consensus pour valider les découpages auprès de la population sont fragiles, la contestation étant favorisée par un contexte accru de liberté et de diversité (régionale, politique, etc.). Cette situation ne faciliterait pas les regroupements et les réunions des personnes d'un même quartier, surtout lorsqu'elles ne voient pas immédiatement leurs intérêts en jeu. Une autre particularité du milieu urbain est l'importance et la diversité de l'offre de soins qui permettent aux populations, en premier lieu aux plus aisées, de choisir elles-mêmes leurs lieux de soins, indépendamment de la carte sanitaire.

Une solution pour pallier cette complexité du découpage en milieu urbain était de faire coïncider ce découpage avec le découpage administratif. Aussi, le découpage de Yaoundé s'est fait sur la base du découpage en arrondissements, alors au nombre de quatre. Le processus s'est poursuivi jusqu'au niveau des aires de santé, où le découpage a respecté l'existence de quartiers et de blocs, en les regroupant le cas échéant. Cette adéquation permettait de restreindre les difficultés de coordination administrative dans un contexte généralisé de décentralisation en faveur des arrondissements urbains.

Aux quatre arrondissements urbains de Yaoundé ont donc d'abord correspondu quatre districts sanitaires. Le maintien de cette adéquation initiale, souhaitée par les autorités sanitaires, s'est heurté à un découpage administratif caractérisé par un processus évolutif encore à l' œuvre après l'instauration des districts sanitaires. Car le découpage administratif a été modifié en 1994, soit trois ans après les études pour la délimitation des districts de santé à Yaoundé et Douala. Les arrondissements à Yaoundé sont passés de quatre à six. Ainsi, le district sanitaire de Biyem Assi, qui initialement était confiné au sein de l'arrondissement de Yaoundé III, englobe désormais deux arrondissements : Yaoundé III et Yaoundé VI, issu d'une partition de l'ancien Yaoundé III.

Les raisons de l'évolution de ]a structuration administrative sont diverses. Depuis une vingtaine d'années, l'Administration territoriale a procédé à une multiplication des échelons administratifs. Le Cameroun est passé ainsi de 7 à 10 provinces. Cette stratégie a permis notamment d'accroître le nombre de postes et la redistribution, et multiplier ainsi les obligés politiques. Or, pour ce qui est du processus de scissiparité, les structurations techniques, sanitaires, ne sont pas toujours aussi dynamiques que les structurations administrativo-politiques. Du fait du caractère pluriel des intervenants, toute modification nécessite du temps, lorsqu'elle ne se révèle pas impossible. 


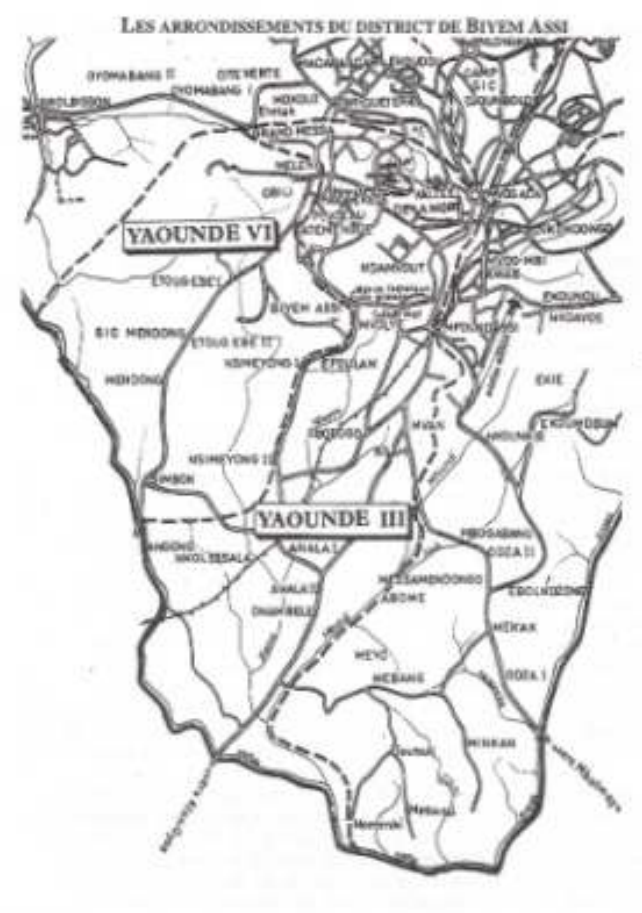

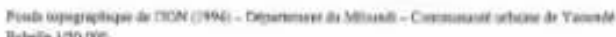
hatele iso of

Les arrondissements du district de Biyem Assi

Voir carte 1 en document annexe administrative et sanitaire, à Yaoundé, par la création de six districts sanitaires à Yaoundé s'est heurté à la réticence du principal bailleur de fonds, la Banque Mondiale, qui n'acceptait pas la remise en cause d'un plan d'intervention qui venait d'être arrêté dans le cadre de son Projet Santé-Fécondité-Nutrition (PSFN). Il est donc resté quatre districts sanitaires pour six arrondissements. Dans ce cas, comment faire correspondre deux schémas d'organisation territoriale discordants, surtout lorsque le modèle de référence est en perpétuelle évolution?

Selon le Chef de Service de Santé du District de Biyem Assi, le fait de rendre compte à deux sous-préfectures n'entraîne pas de problèmes particuliers, ces dernières pouvant collaborer le cas échéant, même s'il est plus facile de "gérer un sous-préfet plutôt que deux". Lors des cérémonies officielles, des problèmes de préséance peuvent en effet surgir entre les deux sous-préfets. Le Service de Santé de District doit alors, le cas échéant, en référer au Préfet. Dans le cas de figure de Biyem Assi, l'historique de la constitution des deux arrondissements permet d'atténuer ce problème de préséance : l'arrondissement de Yaoundé VI est né d'une partition de l'arrondissement de Yaoundé III, le préfet du "grand Yaoundé III" (c'est-à-dire l'arrondissement initial) étant toujours en place à la sous-préfecture du nouvel arrondissement de Yaoundé III, il s'est instauré une relation qualifiée "d'aîné à cadet" entre celui-ci et le sous-préfet de Yaoundé VI, qui est en outre plus jeune que son homologue. En fait, les décrets de création des districts ne précisent pas quelles sont les autorités de tutelle des districts sanitaires sur le plan territorial, du fait de l'hétérogénéité de leurs découpages, créant alors un vide juridique à ce niveau. 

district, car l'autorité administrative qu'est la sous-préfecture gère une certaine catégorie d'agents de l'Etat (catégorie C et D) présents dans les centres de santé. Ainsi le centre de santé de Mendong, qui fait partie d'une aire de santé située dans l'arrondissement de Yaoundé VI, a été construit, de façon inopinée, sur un terrain du quartier de Saint-Siméon, limitrophe des deux arrondissements, mais qui dépend de Yaoundé III. Ceci a entraîné des dysfonctionnements dans la gestion du personnel: quelle était l'autorité administrative responsable de l'affectation et de la notation du personnel de ce centre de santé qui "n'était pas à la bonne place"? Le litige administratif n'a pu être réglé qu'en fermant le centre de santé avant de procéder à sa réimplantation ultérieure.

fance pas également de problèmes particuliers, même si elle se révèle complexe. En fonction du découpage géographique de l'Administration territoriale, le responsable du service de santé du district se réfèrera en fonction des circonstances au sous-préfet de Yaoundé III ou de Yaoundé VI, pour ce qui est du district de Biyem Assi. Cependant, il reconnaît que "avoir à jongler entre les deux sous-préfets" pose le problème d'une identification claire de l'interlocuteur administratif. Lorsque le responsable du district doit solliciter l'autorité administrative pour une réunion, à quel sous-préfet doit-il s'adresser? Un exemple particulièrement révélateur est donné à Douala où un district de santé se retrouve à cheval sur une partie de deux arrondissements contigus. Le sous-préfet a alors affaire à deux chefs de santé de district, qui eux-mêmes dépendent de deux sous-préfets différents. Cela pose aussi des problèmes de représentation pour le chef de santé de district qui doit être présent aux différentes réunions organisées par les deux sous préfets. Cette situation peut être d'autant plus problématique que, si le responsable du district a la compétence technique pour organiser les réunions, il n'a pas l'autorité pour convoquer les chefs de quartier et de village des arrondissements, prérogative du sous-préfet de l'arrondissement et du gouverneur de la province. Cela pose aussi des problèmes de coordination puisque l'autorité administrative est supposée représenter le gouvernement au niveau local, et est donc chargée de la coordination des services administratifs implantés au sein de sa compétence territoriale. Dans l'exemple de Biyem Assi, l'autorité administrative pour le district de santé est double avec les deux sous-préfectures de Yaoundé III et Yaoundé VI. Or, sous quelle autorité administrative le Service de Santé de District doit-il coordonner les activités de santé dans ce district sanitaire? Est-ce le sous-préfet de Yaoundé III ou celui de Yaoundé VI ? A moins de considérer une partition de facto sinon de jure entre les formations sanitaires qui seraient $\mathrm{du}$ ressort territorial et tutélaire de la sous-préfecture de Yaoundé III et de la sous-préfecture de Yaoundé VI. Cette question est d'autant plus importante que la santé publique met l'accent sur l'intégration des activités de santé, avec la participation des autres secteurs comme l'éducation, l'hygiène, l'information, l'agriculture, etc.

La constitution du district de Biyem Assi Le district

Situé au Sud-Ouest de Yaoundé, le district sanitaire de Biyem Assi couvre deux arrondissements de la ville (arrondissements III et VI); les estimations oscillent en 1999, selon les sources, entre 187.536 habitants, pour les services de santé de district, et près de 315.564 pour les autorités administratives; près de $25 \%$ de la population de Yaoundé y résiderait. Une grande diversité caractérise le district de Biyem Assi : zone 
rurale, quartiers d'habitats précaires, quartiers intermédiaires, zones résidentielles. Des zones dites d'habitats spontanés se caractérisent par des constructions ne bénéficiant pas d'adduction d'eau. L'arrondissement de Yaoundé III bénéficie du projet de la Mission d'Aménagement des Terrains Urbains (MAETUR), chargée du lotissement et de la construction de logements collectifs à des prix abordables. On retrouve dans ce quartier les plus pauvres comme les plus nantis, avec des activités professionnelles très diversifiées ; une partie importante de la population travaille dans la fonction publique, d'autres dans les secteurs privé et informel, ou dans l'agriculture. La population est très cosmopolite, pratiquement toutes les communautés culturelles du pays y sont représentées, et elles s'organisent volontiers en associations à caractère socioculturel. Des entreprises privées et parapubliques sont installées sur le territoire du district sanitaire de Biyem Assi : les Brasseries du Cameroun, la Société de publication et d'édition du Cameroun, SODEPA, SCDP et diverses petites entreprises privées dans des secteurs aussi variés que la menuiserie, la peinture et l'agro-alimentaire. Précisons également que l'université de Yaoundé 1 est située dans ce district. On y dénombre 24 "quartiers-villages"; un chef de quartier se trouve à la tête de chacun de ces "quartiers-villages".

25 La création du district sanitaire a eu lieu en 1994. Le district de Biyem Assi a été subdivisé en 10 aires de santé. La population par aire de santé varie de 7.103 habitants (aire de santé d'Afanoya) à 59.059 habitants (aire de santé de Melen). Pour ce qui est du découpage en aires de santé, les négociations se sont déroulées entre les autorités sanitaires, administratives et celles du Projet Santé-Fécondité-Nutrition soutenues par la Banque Mondiale. Le découpage semble avoir tenu compte de l'existence des quartiers tels qu'ils sont nommés par les habitants et des formations sanitaires préexistantes, le cas échéant.

26 Avant ce découpage sanitaire, chaque arrondissement de la capitale possédait son Centre Médical d'Arrondissement (CMA). La restructuration de la ville en districts de santé a nécessité un ajustement des fonctions des formations sanitaires existantes. Ainsi, pour la zone de Biyem Assi, le CMA d'Efoulan se trouve dorénavant défini comme un Centre de Santé Intégré (CSI) alors que son plateau technique le situerait plutôt comme une structure de référence du district de santé. L'hôpital de district, nouvellement construit dans l'arrondissement VI, couvre les deux arrondissements concernés (Yaoundé III et VI). L'équipe cadre du district se trouve basée au CSI de Mendong, dans l'arrondissement de Yaoundé III.

Plusieurs formations sanitaires sont installées dans le district de Biyem Assi. Il est difficile de les classer étant donné la diversité de leurs dénominations, de leurs statuts et de leurs fonctions. Les formations sanitaires publiques se situent à deux niveaux de la pyramide sanitaire : le niveau central (CHU; hôpital militaire de la Garnison) et le niveau périphérique. Trois types de formations sanitaires appartiennent au niveau périphérique: les centres de santé intégrés, le centre médical d'arrondissement et l'hôpital de district. Certaines formations sanitaires publiques ne sont pas gérées par le ministère de la Santé, comme l'hôpital militaire, les infirmeries de l'armée (ministère de la Défense), le Centre national des handicapés (ministère des Affaires sociales), et le centre médico-universitaire (ministère de l'Enseignement supérieur). La situation des formations sanitaires publiques du district est résumée dans le tableau ci-dessous : 
Tableau 1 : Les structures publiques du district de Biyem Assi

\begin{tabular}{|c|c|c|c|c|}
\hline & \multicolumn{4}{|c|}{ Ministères de rattachement } \\
\hline Niveaux & $\begin{array}{l}\text { Santé } \\
\text { publique }\end{array}$ & Affaires sociales & Défense & $\begin{array}{l}\text { Enseignement } \\
\text { Supérieur }\end{array}$ \\
\hline Central & $\mathrm{CHU}$ & $\begin{array}{l}\text { Centre national des } \\
\text { handicapés }\end{array}$ & Hôpital militaire & $\mathrm{CHU}$ \\
\hline Périphérique & $\begin{array}{l}\text { Hôpital de } \\
\text { district } \\
\text { CMA } \\
\text { d'Efoulan } \\
\text { CSI d'Ahala } \\
\text { CSI d'Etoa } \\
\text { Yeyap de } \\
\text { CSI } \\
\text { Mendong }\end{array}$ & & $\begin{array}{l}\text { Infirmerie de la Garde } \\
\text { présidentielle } \\
\text { Infirmerie du Camp } \\
\text { Yeyap }\end{array}$ & $\begin{array}{l}\text { Centre médical } \\
\text { universitaire }\end{array}$ \\
\hline
\end{tabular}

\section{District de Santé de Biyem Assi}

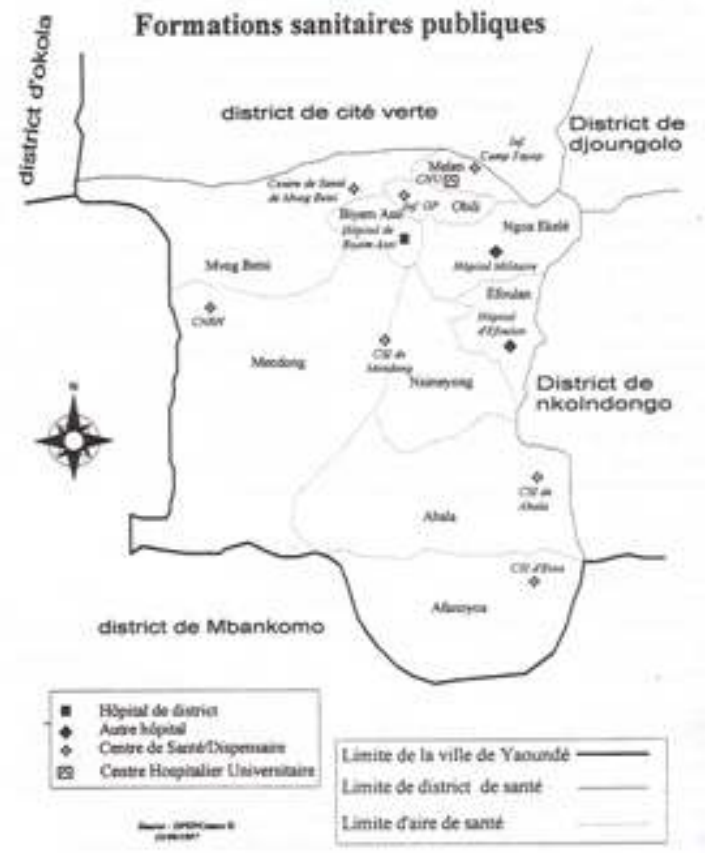

District de santé de Biyem Assi - Formations sanitaires publiques

Voir carte 2 en document annexe 


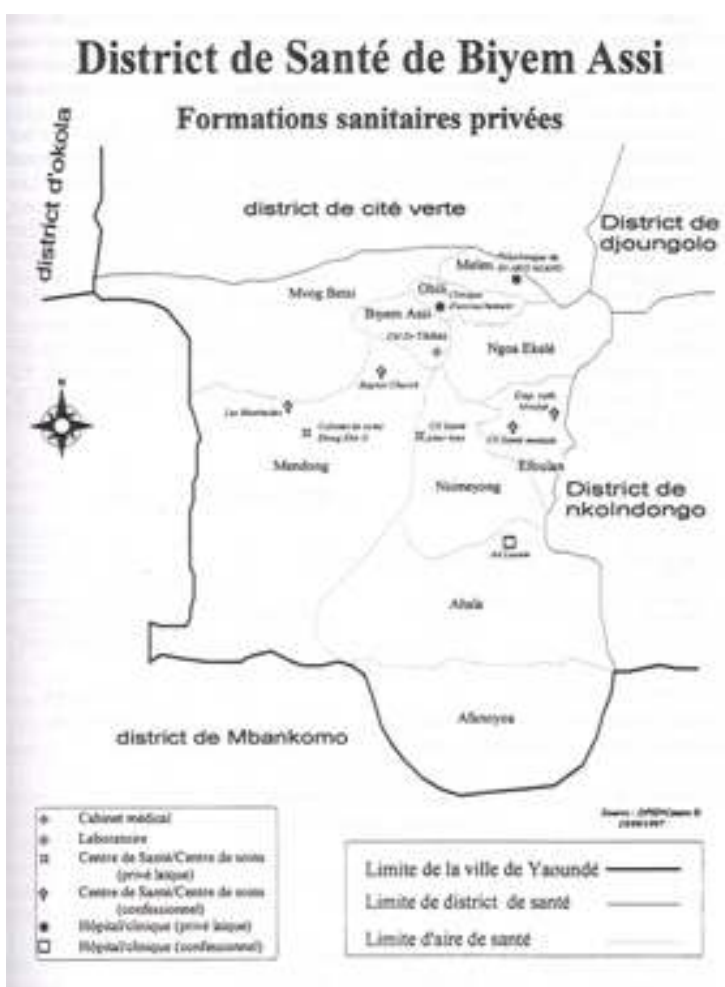

District de santé de Biyem Assi - Formations sanitaires privées

Voir carte 3 en document annexe

Deux hôpitaux de troisième référence (hôpital militaire et $\mathrm{CHU}$ ) sont donc présents dans le district. Ces hôpitaux ne devraient s'occuper que des soins du troisième niveau (référence des hôpitaux régionaux et de districts); dans la pratique, ces établissements reçoivent aussi bien les malades pour les soins tertiaires que primaires, et le service de santé de district n'a aucun droit de regard sur ce type d'établissement.

On trouve également dans le district nombre de formations sanitaires privées à but lucratif ou non. La dénomination des formations sanitaires privées à but lucratif permet de les scinder en deux groupes : les cabinets de soins et les cabinets médicaux. Les cabinets de soins sont dirigés par un infirmier diplômé d'Etat ou un technicien supérieur en soins infirmiers, ils proposent officiellement des activités de soins infirmiers. Les cabinets médicaux sont dirigés par un médecin et doivent proposer des activités médicales autorisées par la loi. Les cabinets de soins et les cabinets médicaux n'ont pas de vocation d'hospitalisation; ils ne peuvent garder les malades qu'en observation journalière. Ces formations sanitaires viennent compliquer particulièrement l'organisation de la couverture sanitaire pour les districts urbains : la plupart d'entre elles ne couvrent pas une zone précise en activités préventives et promotionnelles. Le tableau suivant présente les formations sanitaires privées recensées par le Service de Santé du District ${ }^{2}$.

Tableau 2 : Les structures de soins privés du district de Biyem Assi 


\begin{tabular}{|c|c|c|}
\hline & Cabinet de soins & Cabinets médicaux \\
\hline $\begin{array}{l}\text { Hôpital annexe de Djoungolo } \\
\text { Hôpital Ad Lucem } \\
\text { Baptist Church dispensary } \\
\text { Centre médical Santé mentale } \\
\text { Centre de santé des Béatitudes } \\
\text { Dispensaire catholique de } \\
\text { Mvolyé }\end{array}$ & $\begin{array}{l}\text { Clinique d'accouchement } \\
\text { Fondzenwa } \\
\text { Cabinet de soins Etoug Ebe II } \\
\text { Cabinet de soins chinois } \\
\text { Cabinet de soins Ouanang } \\
\text { Cabinet de soins Indjike } \\
\text { Cabinet de soins Kouagang } \\
\text { Cabinet soins Médecine globale } \\
\text { Cabinet de soins Saint Esprit } \\
\text { Cabinet de soins Sainte Anna } \\
\text { Cabinet de soins Bitchoka } \\
\text { Cabinet de soins Oxygène }\end{array}$ & $\begin{array}{l}\text { Cabinet médical Dr. Timma } \\
\text { Cabinet médical Santé pour } \\
\text { tous } \\
\text { Cabinet médical Besafaca } \\
\text { Cabinet médical Chinois }\end{array}$ \\
\hline
\end{tabular}

L'absence d'étude des bassins de recrutement de chaque formation sanitaire, tant publique que confessionnelle, rend difficile la caractérisation de ce district. Selon le responsable du district, la majeure partie des patients fréquentant les formations sanitaires du district sont originaires de Biyem Assi. En fait, dans les critères de délimitation des districts et des aires de santé élaborés à Bertoua pour la carte sanitaire camerounaise, le critère de l'existence des formations sanitaires n'a pas été corrélé avec celui de leur fréquentation, ni avec celui de leur bassin de recrutement.

Les aires de santé

Dix aires de santé composent le district: Melen, Mvog Betsi, Obili, Biyem Assi proprement dit, Mendong, Ngoa Ekele, Efoulan, Nsimeyong, Ahala, Afanoya. Toutes ne sont pas opérationnelles et ne sont pas desservies par les mêmes types de structures de soins (cf. cartes). Les différentes aires de santé de Biyem Assi illustrent parfaitement la diversité géographique et sociale qui caractérise ce district. Certaines aires desservent des communautés péri-urbaines, voire des communautés totalement rurales, situées à plus de 10 kilomètres du centre du district, et difficiles d'accès; on peut supposer que le comportement de ces populations est fort différent de celui des fonctionnaires de la zone lotie.

Le fonctionnement des aires de santé dépend de la présence opérationnelle ou non d'une structure publique ou privée. Certaines aires sont dépourvues de formation sanitaire de base (Obili, Melen, Ngoa Ekele); cependant, à Melen, les services de santé sont assurés par le $\mathrm{CHU}$, et à Ngoa Ekele, par l'hôpital militaire. Ces structures de référence nationale qui desservent de fait des aires de santé sont présentes à côté de la formation sanitaire de district nouvellement construite. La présence de ces hôpitaux inciterait la population à s'implanter dans ces arrondissements, selon les élus. D'autres aires de santé sont équipées de deux CSI (Afanoya). Nombre d'aires de santé sont également desservies par des formations sanitaires privées. Il semblerait que certaines de ces formations restent dans l'attente d'un agrément du ministère de la Santé. Disposant d'une clientèle fidélisée, l'absence de cet accord ne les empêche pas de fonctionner. Les formations sanitaires confessionnelles ont un bassin de desserte 
s'étendant bien au-delà des limites des aires de santé. Selon l'équipe de district, seules quelques formations privées suivent la politique de santé du district.

Chaque centre desservant une aire de santé est censé appliquer une politique de recouvrement des coûts, sous la supervision d'un comité de santé, qui doit notamment permettre l'approvisionnement en médicaments. Cependant, certains centres, à l'exemple de celui d'Etoa, vivent de dons en produits pharmaceutiques. D'autres formations sanitaires, à l'exemple du centre d'Ahala, nouvellement construit, restent dans l'attente d'une avance en médicaments et de la constitution d'un comité de santé afin de pouvoir démarrer une politique de recouvrement des coûts.

Un des reproches formulés par nos interlocuteurs est l'absence de suivi technique des différentes formations sanitaires par l'équipe-cadre du district, notamment en matière de supervision par le comité de santé du district du personnel sanitaire affecté dans ces centres; d'une manière générale, ils soulignent l'absence d'une politique en santé cohérente dans ce district. Il n'y a guère de contact entre les personnels des centres périphériques et l'équipe du district hormis lors des journées de vaccination, et la collaboration intersectorielle avec les secteurs apparentés à la santé (Affaires sociales, Education, etc.) n'était pas encore organisée en 1999. Pour certaines aires de santé, qu'il $\mathrm{y}$ ait un district sanitaire ou non, les problèmes restent entiers : "le gouvernement ne fait rien en dehors de l'affectation du personnel; on ne voit pas les faits", selon un médecin exerçant dans une aire de santé semi-rurale du district.

Prenons l'exemple de l'aire de santé d'Afanoya, la plus excentrée du district et qui couvre les villages d'Etoa, où est implanté le centre de santé, d'Afanou et de Ntuessong. La population est estimée à 7.000 habitants. L'aire de santé créée en 1995 comprend 5 villages desservant chacun une zone de santé. A Etoa, le centre de santé ne date que de 1987. Auparavant, les habitants de ce village allaient se faire soigner au centre médical d'arrondissement d'Efoulan ou au centre de santé de Ntuessong qui fait partie de la même aire de santé. Le centre de santé d'Etoa est situé à 12 kilomètres des quartiers centraux de Yaoundé, le centre de santé de Ntuessong est distant de 15 kilomètres environ de la capitale. Le centre de santé d'Etoa est dirigé depuis l'origine par un infirmier breveté. Mais il faut compter avec la présence d'un médecin retraité qui a joué un rôle important dans l'évolution du système de santé du Cameroun.

Ce médecin fut, entre autres, chef du service départemental de la santé publique dans la province de l'Est, médecin-chef de l'hôpital d'arrondissement de Buea, Délégué provincial de la Santé publique de la province du Sud-Ouest, Délégué provincial du Centre-Sud puis Délégué provincial du Centre après la partition de la province du Centre-Sud. Il prend sa retraite en 1988 et est à l'origine de la construction du centre de santé d'Etoa par l'Etat ; il est originaire d'un village situé dans cette aire de santé. Il est omniprésent au centre de santé d'Etoa, il participe au bon fonctionnement de celui-ci en fournissant de temps en temps des médicaments et du petit matériel, aidé parfois en cela directement par des ONG du Nord. Il consulte même à l'occasion, suppléant alors l'infirmier breveté qui dirige ce centre. Au sein du district de Biyem Assi, il se reconnaît une légitimité certaine : "Dans tout le district, je ne vois pas de médecin aussi âgé que moi, ayant autant d'expérience et étant à la retraite ; je suis leur aîné !" C'est à Etoa qu'il peut encore le mieux ressentir l'importance qu'il conserve d'un passé de dimension nationale. Cependant, il manifestait également beaucoup d'intérêt pour la création du comité de gestion du district dont il aurait aimé être membre. Le seul frein à cette participation, disait-il alors, pourraient être les jeux politiques qui ne 
manqueraient pas de se manifester à l'occasion de l'élection des membres du comité de gestion. Un autre sujet d'inquiétude, pour lui, était de savoir si le rôle du président du comité de gestion de district, dans lequel il se serait bien vu, aurait la même audience que celui de président d'un comité de gestion d'un centre de santé comme celui d'Etoa. En d'autres termes, nous avons là l'exemple d'un centre périphérique très excentré, situé dans une zone semi-rurale, mais dont l'animateur (officieux) principal pourrait être qualifié "d'élite" au sens camerounais du terme, c'est-à-dire un personnage à l'entregent national, avec des connections internationales, et qui finalement peut faire en sorte que le centre de santé soit peu intégré dans le district sanitaire dont il fait partie.

L'action de la Banque Mondiale dans la mise en place des districts à Yaoundé

37 Le financement de la Banque Mondiale se distingue des financements des autres bailleurs qui appuient la réforme de la politique sanitaire, en ce que cet organisme multilatéral ne propose ni des aides directes, ni une assistance technique, mais des prêts à très faible taux pour la réalisation de programmes. Seul l'Etat, sous le contrôle gestionnaire de la Banque Mondiale, est chargé de la mise en œuvre. Le gouvernement camerounais a donc contracté un prêt auprès de la Banque Mondiale pour financer la mise en œuvre des districts de santé au Cameroun, dans le cadre du "Projet Santé Fécondité Nutrition" (PSFN). Le Projet devait durer initialement 60 mois, avec un coût total estimé à 240 millions de FF. Il prévoyait des constructions et des rénovations de bâtiments, l'achat du matériel et des médicaments, la formation du personnel et des représentants de la communauté, ainsi que la mobilisation sociale. Le ministère de la Santé était l'organe d'exécution, l'équipe de coordination du projet, la Banque se limitant à donner un appui technique, logistique et financier.

Des difficultés de fonctionnement se sont manifestées à plusieurs niveaux. D'abord le pouvoir de décision opérationnel est resté très centralisé : pour la moindre activité et le moindre décaissement, l'équipe du projet devait soumettre le dossier au ministre qui, une fois la décision prise, devait obtenir une "non-objection" de la Banque Mondiale à Washington. Les personnels du ministère de la Santé qui travaillaient dans les institutions d'exécution du projet (district, délégation provinciale) considéraient par ailleurs qu'ils gagnaient personnellement peu contrairement à l'équipe de coordination du projet, estimant alors que les résultats devaient être du seul ressort de l'équipe de coordination. Enfin, l'importance des montants, les intérêts et les responsabilités qui en découlaient ont conduit à la mise en place de structures administratives de contrôle et de gestion très lourdes. Ainsi une commission constituée de représentants de plusieurs ministères, des représentants de la Présidence de la République et du Premier ministre présidait à la supervision bi-hebdomadaire du projet.

Initialement, il avait été convenu que la Banque Mondiale soutienne la création des quatre districts de Yaoundé, des cinq districts de Douala, ainsi que de neuf districts ruraux répartis dans cinq provinces différentes; les districts ruraux alors retenus, délaissés par les autres bailleurs impliqués dans la réforme du système de santé, étaient particulièrement difficiles d'accès: à l'Extrême-Nord du pays, et aux confins des provinces de l'Est et de l'Ouest. La Banque Mondiale entendait développer ces districts dans leur globalité, à savoir construire ou réhabiliter toutes les formations sanitaires, construire l'hôpital de district en cas de besoin, ainsi que le Service de santé de district. Globalement, il s'agissait d'un programme concernant 150 centres de santé, 18 hôpitaux de district et 18 Services de santé de district. 

bureaucratie des Nations Unies allait considérablement ralentir le projet. L'exemple du recrutement du "consultant international en formation" permet d'illustrer ces lenteurs administratives. Dans la programmation initiale du projet, la Banque Mondiale avait exigé que la cellule de la formation soit assistée par un consultant international pour la préparation de la formation du personnel. Le processus de recrutement de ce consultant a mobilisé toutes les parties concernées pendant un an sans aboutir. Pour éviter de cumuler du retard, la cellule de la formation a organisé une série de séminaires-ateliers avec des formateurs de divers horizons (Faculté de médecine de Yaoundé, organismes de coopération, Ministère de la Santé Publique, délégations provinciales, districts, etc.) - et le travail de préparation des formations a été conduit avec succès puisque le représentant de la Banque Mondiale avait reconnu sa pertinence. Mais il fallait attendre la fin du processus de recrutement de cet expert, car la Banque Mondiale exigeait qu'il vienne valider le travail fait par des nationaux.

Devant les retards accumulés, la Banque Mondiale a décidé en 1999 de restreindre son champ d'intervention à un seul district à Yaoundé et à Douala ; à Yaoundé, le district retenu est celui de Biyem Assi, à Douala, celui de New Bell; et sur les neuf districts ruraux initiaux, seuls trois seront maintenus après la revue du projet en mai 1999.

Les raisons du choix de Biyem Assi comme district urbain soutenu par la Banque Mondiale à Yaoundé sont difficiles à apprécier. Les principaux responsables du ministère de la Santé impliqués dans le projet de santé urbaine ne peuvent expliquer ce choix, pas plus que celui du district New Bell de Douala, car ils n'ont pas été consultés. D'ailleurs, pour ces responsables, le choix de New Bell à Douala est contestable car, pour assurer le succès d'un projet-pilote en milieu urbain il vaut mieux l'implanter à l'endroit où il trouve les meilleurs chances de réussite, pour avoir le temps d'améliorer le projet et de le répliquer. Or, le district de New Bell est l'une des zones les plus insalubres de la ville; il souffre d'un manque d'espace pour construire de nouveaux centres de santé ; les "structures de dialogue" pour la participation communautaire y sont absentes; il n'y a pas de voies de desserte fonctionnelle pour se rendre à l'hôpital de district, notamment en cas de pluie où les rares routes deviennent des bourbiers. Selon un expert du ministère de la Santé, le choix de poursuivre le développement des districts de Biyem Assi et de New Bell s'expliquerait par le fait que les activités avaient déjà commencé dans ces deux districts, notamment pour ce qui est des travaux de réhabilitation et des activités de formation du personnel. De plus Biyem Assi disposait déjà d'un hôpital et de l'espace nécessaire autour pour l'agrandir. Comme le programme devait commencer par des constructions et les travaux de réhabilitation des hôpitaux de district, Biyem Assi se distinguait donc par son espace et ses possibilités d'agrandissement. De plus, sur les quatre districts sanitaires de Yaoundé, deux seulement étaient déjà dotés d'un hôpital, à savoir Biyem Assi et la Cité Verte. Pour ce dernier, l'espace était très réduit et ne permettait pas facilement d'y incorporer les différents aménagements nécessaires aux fonctions minimales définies pour les nouvelles normes d'un hôpital de district.

Pour un Directeur central du ministère de la Santé, un tel projet de la Banque Mondiale à Yaoundé avait des atouts importants : le ministère disposait du "Cadre conceptuel d'un district de santé viable au Cameroun"3, document de base pour les différents acteurs de cette réforme, le ministère de la Santé disposait également des ressources humaines nécessaires à l'implantation du district sur le terrain; les communautés 
avaient déjà fait l'objet d'une sensibilisation en partenariat avec le ministère de la Santé ; et surtout le financement de la Banque Mondiale était disponible. Cependant, il déplore le manque de coordination qui s'est alors traduit par une gestion au jour le jour du projet. Ce n'est qu'en 1998, soit trois ans après le démarrage du projet, avec une nouvelle équipe au ministère de la Santé, qu'un plan d'action pour les années à venir a été élaboré. Par ailleurs, la traduction des décisions du ministère sur le terrain était difficile, notamment du fait de la lourdeur de la conception globale du district de santé qui nécessitait l'apport de nouvelles compétences qui ne trouvent pas toujours leur compte dans le projet et restreignent alors leur implication. Ce projet a souffert aussi des clauses de sa mise en œuvre qui était axée sur une décentralisation maximale. Plus précisément, dans ce projet, se retrouvaient impliqués des acteurs situés aux trois niveaux (ministère, province, district) de la pyramide sanitaire définie par la REO des SSP. La collaboration entre le niveau central et l'unité de coordination du projet a été difficile à obtenir, les différentes Directions du ministère n'étant pas toutes impliquées de la même manière dans le programme (ainsi de la Direction de la Pharmacie et des Médicaments, pour les médicaments essentiels, ou de la Direction des Ressources Humaines, pour les affectations du personnel). Une des réorientations des modalités des relations avec l'unité de coordination de l'ensemble du programme a consisté à choisir des pôles préférentiels de collaboration au sein des Directions centrales. On peut se demander enfin si une intervention externe de cette envergure n'a pas eu également un rôle déstructurant; à force de promesses (approvisionnement en médicaments, budgets accordés aux comités de santé), elle a fait naître beaucoup d'espoir, éventuellement exacerber les rivalités pour l'accession aux postes représentatifs, comme la participation aux comités de santé ou de gestion, dont on supposait qu'ils pouvaient être particulièrement rémunérateurs.

Faire exister le niveau intermédiaire à YaoundéLa Délégation provinciale de la santé Nominalement, le Délégué provincial est le représentant technique du ministre de la Santé dans la province, principe bien souvent contredit dans la réalité à Yaoundé, car la proximité avec les instances du ministère de la Santé fait que ce délégué représente quelqu'un de puissant déjà présent dans la ville. Cette situation a deux conséquences majeures : une pression accrue du niveau central, et un accès direct du niveau local au niveau central, le niveau intermédiaire de la province étant alors contourné. La tentation est offerte de passer par-dessus le Délégué provincial pour aller rencontrer directement les responsables centraux du ministère ; à l'inverse des services centraux $\mathrm{du}$ ministère peuvent contourner le niveau intermédiaire pour des décisions concernant la santé publique dans un quartier de Yaoundé. Ainsi, la nomination du nouveau médecin-chef de l'hôpital de district de Biyem Assi et la date de sa prise de fonction, en 1999, furent ignorées par le Délégué provincial.

La Délégation provinciale est aussi fragilisée par le fait que toutes les formations sanitaires présentes dans la province du Centre, ne sont pas de son ressort. C'est le cas de l'hôpital central et de l'hôpital général de Yaoundé, hôpitaux de troisième ligne relevant directement du niveau central et non de la province; ne relèvent de la Délégation provinciale que les hôpitaux de district, les centres médicaux d'arrondissement (CMA) et les centres de santé. Le fait que des établissements de soins ne fassent pas partie de la carte sanitaire prévue par la réforme a entre autres conséquences une gestion particulièrement difficile de l'information sanitaire provinciale. Par ailleurs, le Délégué provincial n'a pas la capacité d'affecter du personnel de l'hôpital central dans les districts, ou inversement, en vue d'un 
redéploiement interne à la province du Centre. Le Délégué n'a prise que sur les personnels de district. Dans le même temps, il est dans l'incapacité de déterminer le ratio des personnels des établissements publics et parapublics sanitaires par rapport à la population de la province, car ne disposant pas des informations sur les ressources humaines de l'ensemble des structures de soins de sa province. Enfin, il ne connaît guère les profils pathologiques et épidémiologiques des patients qui se rendent dans ces hôpitaux qui ne relèvent pas de sa compétence territoriale. Comment alors organiser le système de référence / contre référence dans ce cas?

Pour remédier à cette situation, il a été envisagé de mieux structurer la référence en attribuant des compétences territoriales, en quelque sorte, à chacun des grands établissements hospitaliers de Douala et de Yaoundé. Ainsi, l'hôpital central de Yaoundé deviendrait la référence de la province du Centre et assurerait le parrainage de tous les hôpitaux de district de cette province ; la province du Sud relèverait aussi de la compétence de l'hôpital central, alors que les provinces du nord du pays seraient desservies par l'hôpital général de Yaoundé, et les provinces de l'ouest, par l'hôpital général de Douala. Ce projet de complémentarité des formations sanitaires de la province du Centre dont font partie tous les établissements publics de Yaoundé, serait mené sous l'égide conjointe de la Délégation provinciale et de l'hôpital central, ce dernier n'entrant pas dans le giron de la Délégation provinciale mais allant vers une collaboration plus poussée avec le niveau provincial.

Actuellement, le renforcement ou non de la Délégation en matière de gestion du personnel ou des ressources financières, repose d'abord sur l'évolution économique du pays pour que la décentralisation puisse être effective. Or, les allocations financières de l'Etat mises à la disposition de la Délégation de la province du Centre pour son fonctionnement sont allées en décroissant pendant plusieurs années. Dans le même temps, la Délégation ne bénéficiait toujours pas d'une complète autonomie de gestion, même dans le cadre de contacts directs établis avec les bailleurs de fonds étrangers. En effet, l'accord du niveau central était encore exigé à partir d'un certain niveau d'aide directe des bailleurs aux structures de soins.

Au bout du compte, il semble, de l'avis même d'un ancien directeur provincial de la santé en exercice avant la mise en place de la réforme, que la décentralisation actuelle ait réduit la marge de manœuvre du niveau provincial. Auparavant, le directeur provincial disposait de moyens plus importants en provenance de l'Etat pour acheter directement lits, médicaments, etc., pour équiper les hôpitaux de la province. Il avait également compétence pour les affectations du personnel au niveau de la province, et pour recruter du personnel non qualifié (manœuvres, manutentionnaires, personnels d'entretien, etc.). Il avait même un rôle plus important qu'un directeur central de l'époque qui devait alors passer par l'intermédiaire du directeur provincial s'il voulait réussir dans son action; et, manifestation suprême de l'importance du directeur provincial, celui-ci était au côté du ministre lorsqu'il se déplaçait en province. Cette évocation du "bon vieux temps" où le directeur provincial avait tout pouvoir en tant qu'élite locale reste sans doute en partie vérifiable pour certains délégués provinciaux actuels en milieu rural, mais ne se rencontre pas encore pour le milieu urbain spécifique que constitue la capitale.

Le Service de Santé du District (SSD)

49 La trop grande proximité avec les services centraux est une des principales causes invoquées pour expliquer que le district de Biyem Assi soit en phase perpétuelle de 
démarrage depuis sa création en 1994. Il s'ensuivrait, de la part de l'équipe de district, frustrations et attentes interminables des décisions du niveau central permettant d'avancer dans le développement du district. Les districts ruraux auraient pour leur part davantage progressé du fait d'une moindre prégnance de cette tutelle.

Jusqu'en mai 1999, le Service de Santé de District (SSD) de Biyem Assi a ainsi évolué de façon informelle dans le cadre d'une équipe resserrée autour des quelques collaborateurs du Chef de Service de Santé de District (CSSD), à savoir le Chef du Bureau Santé, le Chef du Bureau des Affaires administratives et financières, et le Médecin Chef de l'hôpital de district de Biyem Assi. Une telle structure ne pouvait pas toujours répondre aux attentes, en particulier de la communauté. Aussi, une équipe-cadre renforcée a été composée pour le district conformément aux directives issues du "Cadre conceptuel d'un district de santé viable au Cameroun", élaboré par le ministère de la Santé. La création d'une telle équipe, dans le contexte de la Réorganisation des Soins de Santé Primaires, doit être formalisée par une note du ministère.

Définie en mai 1999, l'équipe cadre regroupe treize personnes :

- le Chef de Service de Santé de District (CSSD)

- le Chef du Bureau Santé

- le Chef du Bureau Affaires administratives et financières

- le Médecin Chef de l'hôpital de district

- le surveillant général de l'hôpital de district

- un médecin choisi parmi les neuf exerçant au sein de l'hôpital de district

- le Médecin Chef du Centre médical d'arrondissement d'Efoulan

- le surveillant général du Centre médical d'arrondissement d'Efoulan

- un médecin du Centre médical d'arrondissement d'Efoulan

- tous les responsables des Centre de Santé Intégrés de Biyem Assi (3 en août 1999)

Toutes les latitudes offertes par le texte ont été exploitées pour composer cette équipe. En dehors du "noyau dur" formé par les membres titulaires que sont le CSSD, les deux chefs de bureau, le médecin chef et le surveillant général de l'hôpital de district, ont été également désignés des médecins des structures de santé du district qui s'intéressent à la santé publique. De plus, il est préconisé dans le "Cadre conceptuel" d'adjoindre des membres des "secteurs apparentés concernés par le développement de la politique de santé dans le district" ; c'est donc une équipe cadre d'une vingtaine de personnes qui pourrait être constituée à terme. Or, cette marge d'appréciation offerte à chaque Service de Santé de District de procéder à l'établissement de son équipe cadre n'est pas sans susciter interrogations, discussions et remises en cause, comme le montre le caractère provisoire de la première équipe-cadre mise en place à Biyem Assi.

La décentralisation ne concerne pas encore les ressources humaines. Les affectations relèvent de ('autorité administrative pour la décision mais des structures locales pour les propositions. Par exemple, le CSSD doit envoyer ses propositions de redéploiement de personnel à l'intérieur de son district au Gouverneur de la province pour que celui-ci les entérine. Au Gouverneur de la province et non au sous-Préfet de l'arrondissement, car seul le premier a le rang de directeur de l'Administration territoriale et à ce titre a compétence pour affecter le personnel. Le Délégué provincial intervient quant à lui pour les affectations inter-district; le CSSD ne peut muter son personnel en dehors de son district, et là encore c'est le Gouverneur qui intervient en dernière instance : le Délégué provincial prépare les mutations et les envoie au Gouverneur pour qu'elles soient entérinées. 

contrairement aux structures sanitaires comme l'hôpital de district par exemple. Il reçoit pour son fonctionnement une dotation budgétaire du ministère de la Santé. Il rend compte de cet usage à la hiérarchie sanitaire ou aux responsables du ministère des Finances, qui met à sa disposition les fonds. Afin de disposer d'un minimum d'autonomie financière, il conviendrait que soit mis en place un comité de gestion de district où la communauté serait représentée. Le blocage lié à l'absence d'une "structure de dialogue" est préjudiciable à la capacité d'action et à la légitimité du district. A cet égard, la question de la légitimité du service de santé de district se pose d'autant plus que la carte sanitaire place sous sa tutelle le directeur de l'hôpital de district qui, lui, grâce au système de recouvrement, peut disposer des recettes de sa structure, et recruter du personnel auxiliaire. Dès lors, que peut faire un service de santé de district, en haut de la hiérarchie locale, mais "pauvre" et non autonome, face à un hôpital de district qui lui est normalement subordonné mais qui a la possibilité de faire des recettes et de les utiliser à son seul profit? D'ailleurs, des conflits de compétences et de pouvoir ont pu naître entre le médecin chef de l'hôpital de district et le chef du service de santé du district, et qui ont été résolu, à Biyem Assi, en attribuant les deux fonctions à une seule et même personne.

Vers une décentralisation des ressources : l'exemple du médicament produisent, depuis un arrêté ministériel de septembre 1998 qui fixe les conditions d'organisation et de fonctionnement des structures des comités de santé pour les aires et les districts de santé. Cette mesure, que certains qualifient de "révolution", a été prise à l'issue d'un long processus entamé en 1990 avec la loi sur les libertés et illustre la volonté de l'Etat de réaliser effectivement la décentralisation. Cette mesure interrompt le principe de l'unicité des revenus de l'Etat, et induit une plus grande responsabilisation au niveau local; c'est pour cela que la constitution des comités de santé est un pré-requis pour la gestion de ces recettes. L'affectation des recettes devra ainsi être co-signer par le responsable de la formation sanitaire et par le responsable du comité de santé. La décentralisation des ressources est valable aussi bien pour les centres de santé que pour les hôpitaux de district.

capectif visé est également de réduire les capacités d'intervention directe du niveau central qui peut alors se concentrer sur la définition et la conception des politiques à mener, sur les cadres juridiques et réglementaires, plutôt que de se disperser dans la gestion quotidienne des choses et des biens. L'objectif ultime, selon un ancien ministre de la Santé, est d'aboutir à un financement du district qui ne dépendrait plus directement du budget de l'Etat ; cette mesure doit avoir pour vertu de lutter contre la déperdition, l'opacité et les malversations constatées à chaque niveau administratif, qu'il soit ministériel, provincial ou local. Le niveau périphérique ayant toute autonomie et étant désormais responsable, seul le ministère des Finances aurait à exercer une fonction de contrôle, le ministère de la Santé n'intervenant plus à cet égard. L'évolution la plus manifeste dans ce domaine est le passage du don des médicaments dans les formations sanitaires grâce au budget de l'Etat à une autonomie de gestion de chacune des structures de ses recettes, notamment pour doter les structures d'une pharmacie. Et les difficultés de mise en œuvre d'apparaître alors dans ce domaine avec beaucoup d'acuité. 
58 La politique du médicament a longtemps été caractérisée par la gratuité au Cameroun. Pour autant, des systèmes alternatifs ont pu voir le jour comme avec le système des pro-pharmacies dans le milieu des années 1970. Les mairies attribuaient une dotation financière aux médecins des centres de santé, qui achetaient alors les médicaments et les revendaient à bas prix. Les recettes devaient servir normalement à réapprovisionner le stock de médicaments. Cette expérience, qui n'eut pas de suite, ne concernait que les centres de santé. En effet, dans les hôpitaux, il aurait fallu distinguer les médicaments de la pro-pharmacie de ceux de l'hôpital, ces derniers étant fournis par l'Etat et ne pouvant alors être vendus. De nos jours, cette distinction n'existe plus et toutes les structures de soins vendent leurs médicaments. Dans le cadre de la Réorientation des SSP, si une structure bénéficie d'un don de médicaments, ceux-ci doivent être confiés au comité de santé qui est alors chargé de "gérer" ce don.

Ce système d'utilisation des recettes du recouvrement des coûts désormais instauré pour le réapprovisionnement de la structure en médicaments ne laisse pas d'aiguiser la méfiance de tout un chacun. Les rumeurs de détournement et de revente des médicaments par les personnels de santé à leur profit vont bon train. Mais trouver la clef de répartition adéquate des recettes provenant de la vente des médicaments est une question difficile. Que doit-on faire des médicaments provenant de dons: les fournir gratuitement ou les revendre pour contribuer à alimenter le fonds de roulement du centre? Quelles proportions de la recette reverser en guise de prime aux personnels du centre? Pour le fonctionnement général de la structure? Pour le seul réapprovisionnement en médicament? Pour la rémunération des membres du comité de santé?

La Banque Mondiale devait aider à la mise en place des pharmacies dans les formations sanitaires du district. Les fonds nécessaires ont tardé à venir. En juillet 1999, seul l'hôpital de district de Biyem Assi disposait d'une pharmacie héritée de la période d'avant la réforme lorsqu'il était encore hôpital d'arrondissement. Alors que l'Etat n'intervient plus dans les dotations pharmaceutiques des structures sanitaires, les centres de santé du district, dans l'attente de ce crédit de la Banque Mondiale, ne disposaient pas de médicament, plus de cinq ans après l'instauration du district.

Comités de santé, comités de gestion et "communauté"

61 Dans la réforme, la participation communautaire, l'implication des autorités locales sont la pierre angulaire du projet, à tel point, comme nous l'avons souligné, que l'autonomie financière des structures de santé périphériques, et donc leur financement, en dépend. C'est aussi pour cela qu'il est notamment prévu dans le programme de la Banque Mondiale longuement évoqué un financement particulier pour la formation de ce qu'il est convenu d'appeler les "structures de dialogue", lieux d'expression de la communauté. Les autorités administratives locales ont alors à l'évidence un rôle d'animation essentiel à jouer pour la mise en place de ce dernier niveau essentiel de la pyramide sanitaire Les mairies d'arrondissement ont le rôle de mobiliser les populations. Les maires des communes du ressort du district sanitaire sont membres de droit du comité de gestion de l'hôpital de district. En outre, les mairies peuvent intervenir dans le fonctionnement du district par le biais d'une aide budgétaire directe aux formations sanitaires : depuis 1967, le budget d'une commune ne peut plus être adopté sans que 7\% de celui-ci ne soit alloué au secteur de la santé, ce qui permet notamment de rémunérer certains agents de santé qui dépendent de l'administration locale. Elle pourrait être notamment un facteur de prospérité pour le 
district de santé de Biyem Assi qui recouvrent deux mairies d'arrondissement, mais elle reste dans la réalité très fragile et à l'applicabilité réduite. Car cette mesure, obligatoire dans son inscription, est loin d'être effective. Le budget étant prévisionnel, son exécution dépend de la trésorerie réelle de la mairie et de ses priorités, le tout dans une opacité propice aux manœuvres politiques et aux luttes de personnes. En cas de conflit, c'est l'autorité de tutelle des communes, à savoir le Gouverneur, qui est en droit de trancher, même si ce type de recours est très rarement activé. De plus, la crise économique qu'a traversé le Cameroun a conduit les collectivités locales à réduire de manière drastique leurs budgets et leur marge de manœuvre dans les affectations budgétaires. Aussi, les administrations locales étaient beaucoup plus préoccupées par leur survie que par le développement de leurs administrés. La décentralisation devrait permettre de relancer les activités des comités locaux de développement ainsi que la coordination entre les différents départements pour mettre en place un système de collaboration et d'intervention multisectoriel efficace.

La sous-préfecture d'arrondissement est, de par sa position hiérarchique, davantage impliquée dans la mobilisation des élus locaux et des autorités traditionnelles. Elle est un médiateur incontournable lorsque le Service de Santé de District doit rencontrer une autorité traditionnelle, par exemple. C'est elle qui avise cette dernière de la visite d'un responsable du Service de santé de District. C'est aussi la sous-préfecture qui est chargée d'organiser les élections locales, y compris dans le cadre de la mise en place des comités de santé des centres de santé intégrés. Mais, bien évidemment, toute tentative d'organisation de la communauté est susceptible de créer ou de raviver des rivalités politiques.

L'exemple d'un comité de santé du district qui n'a jamais fonctionné est éclairant à cet égard. Un premier comité de santé avait été composé à l'initiative d'une élite locale en excluant délibérément des médecins habitant la zone et qui participaient bénévolement aux activités du centre de santé. Un conflit s'en est suivi, et il s'est fixé notamment sur la question de la mauvaise gestion du stock de médicaments imputée par le comité de santé aux médecins. Or, dans ce cas de figure, c'est la possibilité de donner des médicaments qui pouvait être à la source de ces accusations. Ce centre bénéficiait en effet de dotations gratuites en médicaments de la part d'une ONG du Nord grâce à l'un des médecins. Certains patients, par ailleurs, continuaient de refuser le nouveau système qui leur demandait de payer consultations et médicaments, d'autant plus, ici, que le médecin pouvait fournir gracieusement les médicaments grâce aux dons dont il bénéficiait. On comprend alors aisément qu'une telle situation pouvait créer la confusion et faire naître toutes les suspicions autour de la gestion des médicaments. De plus, parmi les médecins, on comptait une personnalité influente dans le secteur de la santé et alors à la retraite, ainsi que son épouse, encore en activité dans une Direction centrale du ministère et par ailleurs membre du Bureau politique du parti au pouvoir. Ces derniers devaient entre autres "se mesurer" à un ancien député, membre quant à lui du Comité central du parti au pouvoir, et originaire du village. Le comité de santé a été dissout et un nouveau comité devait être mis en place, avec à sa tête un des médecins, après conciliation entre les différentes élites du village, parce que, nous a-t-on expliqué, ce médecin "est la plus importante personnalité du village puisqu'elle est membre du Bureau politique".

Les hôpitaux de district sont également censés bénéficier du soutien et du contrôle d'un comité de santé et d'un comité de gestion. A Biyem Assi, des comités de gestion, 
composés pour l'essentiel des personnels des structures de santé, ont été instituées pour assister dans la gestion les responsables de l'hôpital de district et du centre médical d'arrondissement d'Efoulan. Le ministère de la Santé accorde des crédits de fonctionnement aux structures sanitaires pour couvrir les coûts récurrents (eau, électricité, téléphone, papeterie, etc.), les recettes générées par la formation sanitaire, c'est-à-dire issues de la participation de la population par le biais du paiement des médicaments et des consultations, servant, entre autres, au réaménagement de l'hôpital, éventuellement au gros œuvre, et au recrutement du personnel d'entretien. Ces dépenses sont supervisées par le comité de gestion de la structure.

En revanche, les tentatives de constitution d'un comité de santé du district de Biyem Assi, où siègeraient des représentants de la population, ont été vaines, ce qui est particulièrement malvenu pour un district pilote de la Banque Mondiale. Face à une première injonction de la Banque Mondiale, et eu égard au faible délai accordé, la sensibilisation par les responsables sanitaires du district pour la constitution d'un comité de santé s'est limitée aux autorités administratives et traditionnelles locales. Le résultat obtenu à partir d'une sélection uniquement par le filtre de ces autorités locales et des responsables des structures sanitaires concernées n'a pas donné satisfaction. Le chef du service de santé du district a alors décidé, en accord avec ses autorités de tutelle, de dissoudre le comité de santé composé à la hâte et de ne pas présenter un comité de santé de district non représentatif pour la revue de la Banque Mondiale.

Les interprétations culturalistes à l'égard du fonctionnement ou non des comités de santé vont souvent bon train. On invoque ici le contexte urbain qui regroupe une population socialement, économiquement et culturellement hétérogène et qui rendrait plus difficile la constitution d'un comité représentatif de la population. Là, si les comités fonctionnent, ce serait en raison des dynamiques "traditionnelles" propres à telle ethnie, qui accepterait mieux l'autorité d'un chef et aurait un sentiment communautaire plus développé. Mais l'on sait par ailleurs que les rivalités politiques, identitaires et communautaires sont loin d'être l'apanage du milieu urbain.

Il est certain cependant que la proximité d'une élite locale avec le pouvoir central, parfois au plus haut niveau de l'Etat, peut singulièrement perturber, en cas de conflit autour d'un centre de santé, toute possibilité de médiation de l'ensemble des niveaux de la pyramide sanitaire, du comité de santé à la délégation provinciale.

Alors, la meilleure conclusion est sans doute celle fournie par un chef du service de santé du district de Biyem Assi. Il qualifie la participation communautaire "d'incontournable", ce qu'elle est effectivement dans la réforme, et comme en témoigne les conditionnalités de la Banque Mondiale qui peut suspendre ou retarder ses financements en l'absence de mise en place des "structures de dialogue". Mais jusqu'à présent, la communauté n'a pas encore été amenée à participer au développement du district de santé. 


\section{NOTES}

1.Le pays regroupe 58 départements et 269 arrondissements pour 14 millions d'habitants. Le Cameroun est aujourd'hui divisé en 135 districts de santé.

2.Cette liste n'est pas exhaustive puisque, officielle, elle ne tient évidemment pas compte du très grand nombre de cabinets illégaux.

3.Ministère de la Santé, Division des Etudes, de la Planification, de l'Information et de l'Informatique : Cadre conceptuel d'un district de santé viable au Cameroun.

République du Cameroun - Banque Mondiale. Février 1999, 57p.

\section{AUTEURS}

\section{ERIC GAUVRIT}

Institut d'Études Politiques, Domaine universitaire BP 10133405 Talence, eric.gauvrit@wanadoo.fr

\section{RAPHAËL OKALLA}

Ministère de la Santé Publique, BP 13690, Yaoundé - Cameroun, rokalla@iccnet.cm 